\title{
NONEXISTENCE OF GLOBAL SOLUTIONS IN TIME FOR REACTION-DIFFUSION SYSTEMS WITH INHOMOGENEOUS TERMS IN CONES
}

\author{
By
}

\author{
Takefumi IgARASHI and Noriaki UMEDA
}

\begin{abstract}
We consider initial-boundary value problems for the reaction-diffusion systems with inhomogeneous terms in cones. In this paper we show the nonexistence of global solutions of the problems in time.
\end{abstract}

\section{Introduction}

We consider nonnegative solutions of initial-boundary value problems for the reaction-diffusion systems of the form

$$
\begin{cases}u_{t}=\Delta u+K_{1}(x, t) v^{p_{1}}, & x \in D, t>0, \\ v_{t}=\Delta v+K_{2}(x, t) u^{p_{2}}, & x \in D, t>0, \\ u(x, t)=v(x, t)=0, & x \in \partial D, t>0 \\ u(x, 0)=u_{0}(x), v(x, 0)=v_{0}(x), & x \in D,\end{cases}
$$

where $p_{1}, p_{2} \geq 1$ with $p_{1} p_{2}>1$. The domain $D$ is a cone in $\mathbf{R}^{N}$, such as

$$
D=\left\{x \in \mathbf{R}^{N} ; x \neq 0 \text { and } x /|x| \in \Omega\right\},
$$

where $\Omega$ is some region on $S^{N-1}$ smooth enough.

The initial data $u_{0}(x)$ and $v_{0}(x)$ are nonnegative, bounded and continuous in $\bar{D}$, and $u_{0}(x)=v_{0}(x)=0$ on $\partial D$. The inhomogeneous terms $K_{i}(i=1,2)$ are nonnegative continuous functions in $D \times(0, \infty)$.

In this paper we denote by $B C$ the set of all bounded continuous functions in $\bar{D}$. The "nontrivial solution" denotes the solution $u$ satisfying $(u, v) \neq \equiv 0$ in $D \times(0, T)$ with some $T>0$, it thus means that $\left(u_{0}, v_{0}\right) \not \equiv 0$ with the condition $\left(u_{0}, v_{0}\right) \in B C$. 
For the Laplace-Beltrami operator with homogeneous Dirichlet boundary condition on $\Omega \in S^{N-1}$, define $\omega_{n}$ as Dirichlet eigenvalues and $\psi_{n}(\theta)$ as the Dirichlet eigenfunctions corresponding to $\omega_{n}$ which is normalized so that

$$
\int_{\Omega} \psi_{n}(\theta) d \theta=1
$$

It is following that

$$
\int_{\Omega} \psi_{m}(\theta) \psi_{n}(\theta) d \theta=0
$$

for $m \neq n$. We introduce the Green's function $G(x, y, t)=G(r, \theta, \rho, \phi, t)$ for the linear heat equation in the cone $D$, where

$$
r=|x|, \quad \rho=|y|, \quad \theta=x /|x| \quad \text { and } \quad \phi=y /|y| \in \Omega
$$

The Green's function is expressed to

$$
G(r, \theta, \rho, \phi, t)=\frac{1}{2 t}(r \rho)^{-(N-2) / 2} \exp \left(-\frac{\rho^{2}+r^{2}}{4 t}\right) \sum_{n=1}^{\infty} I_{v_{n}}\left(\frac{r \rho}{2 t}\right) \psi_{n}(\theta) \psi_{n}(\phi),
$$

where $v_{n}=\left[(N-2)^{2} / 4+\omega_{n}\right]^{1 / 2}$, and $I_{v}$ is the modified Bessel function or

$$
I_{v}(z)=\left(\frac{z}{2}\right)^{v} \sum_{k=0}^{\infty} \frac{(z / 2)^{2 k}}{k ! \Gamma(v+k+1)}
$$

with the Gamma function $\Gamma(z)=\int_{0}^{\infty} s^{z-1} e^{-s} d s$ (see Watson [27, p. 395]).

For our first theorem we shall give the conditions of the inhomogeneous terms $K_{i}(i=1,2)$ as following:

$$
\left.\begin{array}{l}
\text { there exist } C_{U}, \hat{\sigma}_{i} \text { and } \hat{q}_{i} \geq 0 \text { such that } \\
K_{i}(x, t) \leq C_{U}\langle x\rangle^{\hat{\sigma}_{i}}(t+1)^{\hat{q}_{i}} \text { for any } x \in D, t \geq 0,
\end{array}\right\}
$$

where $\langle x\rangle=\left(|x|^{2}+1\right)^{1 / 2}$.

Let $L_{a}^{\infty}$ be a Banach space of $L^{\infty}$-functions in $D$ with the norm

$$
\|\xi\|_{\infty, a} \equiv \operatorname{esssup}_{x \in D}\left(\langle x\rangle^{a}|\xi(x)|\right) .
$$

For $T>0$, set

$$
E_{T}=\left\{(u, v):[0, T] \rightarrow L_{\delta_{1}}^{\infty} \times L_{\delta_{2}}^{\infty} ;\|(u, v)\|_{E_{T}}<\infty\right\}
$$

with the norm

$$
\|(u, v)\|_{E_{T}}:=\sup _{t \in[0, T]}\left\{\|u(t)\|_{\infty, \delta_{1}}+\|v(t)\|_{\infty, \delta_{2}}\right\}
$$


where

$$
\delta_{i}=\frac{\hat{\sigma}_{j} p_{i}+\hat{\sigma}_{i}}{p_{i} p_{j}-1} \quad((i, j)=(1,2),(2,1)) .
$$

It is easily seen that $E_{T}$ is a Banach space.

We begin with stating the existence of the local solution for (1).

THEOREM 1. Assume that $u_{0}, v_{0} \in B C, u_{0} \equiv v_{0} \equiv 0$ on $\partial D$, and $\langle x\rangle^{\delta_{1}} u_{0}(x)$, $\langle x\rangle^{\delta_{2}} v_{0}(x)$ are bounded in $\bar{D}$. Suppose that $K_{i}(x, t)(i=1,2)$ satisfy (6). Then there exists a nonnegative solution $(u, v) \in E_{T}$ which solves (1) in $D \times(0, T)$ for some $T>0$.

For given initial values $\left(u_{0}, v_{0}\right)$, let $T^{*}=T^{*}\left(u_{0}, v_{0}\right)$ be a maximal existence time of the solution of (1). If $T^{*}=\infty$, the solutions are global in time. On the other hand, if $T^{*}<\infty$, then the solutions are not global in time. If the solution blows up in finite time such that

$$
\limsup _{t \rightarrow T^{*}}\|u(\cdot, t)\|_{\infty}+\limsup _{t \rightarrow T^{*}}\|v(\cdot, t)\|_{\infty}=\infty,
$$

then the solution is not global, where $\|\cdot\|_{\infty}$ denotes the $L^{\infty}$-norm with respect to space variable.

For our second theorem we shall define a region $\tilde{D}$ such that

$$
\left.\begin{array}{l}
\text { there exist } k>0 \text { and }\left\{x_{m}\right\}_{m=1}^{\infty} \text { satisfying } 0<\left|x_{m}\right|<\left|x_{m+1}\right|, \\
B\left(x_{m}, k\left|x_{m}\right|\right) \subset \tilde{D} \subset D \text { for any } m, \text { and } \lim _{m \rightarrow \infty}\left|x_{m}\right|=\infty,
\end{array}\right\}
$$

where $B(x, r)$ denotes the ball with radius $r$ centered at $x$. We let the inhomogeneous terms $K_{i}(i=1,2)$ satisfy

$$
\left.\begin{array}{l}
\text { there exist } C_{L}>0, \check{\sigma}_{i}, \check{q}_{i} \geq 0 \text { and } \tilde{D} \text { satisfying (10) such that } \\
K_{i}(x, t) \geq C_{L}|x|^{\check{\sigma}_{i}} t^{\check{q}_{i}} \text { for any } x \in \tilde{D}, t \geq 0 .
\end{array}\right\}
$$

For the theorem we should define $\gamma_{+}$denoting the positive root of the equation $\gamma(\gamma+N-2)=\omega_{1}$

$$
\alpha_{i}=\frac{\left(2+\check{\sigma}_{i}+2 \check{q}_{i}\right)+\left(2+\check{\sigma}_{j}+2 \check{q}_{j}\right) p_{i}}{p_{i} p_{j}-1} \quad((i, j)=(1,2),(2,1)),
$$

and

$$
H_{a}=\left\{\xi \in C(\bar{D}) ; \xi(x) \geq M\langle x\rangle^{-a} \psi_{1}(x /|x|) \text { for } x \in \tilde{D} \text { with some } M>0\right\} .
$$

The main result of this paper is summarized in the following theorem. 
THEOREM 2. Assume that $u_{0}, v_{0} \in B C, u_{0} \equiv v_{0} \equiv 0$ on $\partial D$, and $K_{i}(x, t)$ $(i=1,2)$ satisfy (11). Suppose that one of the following two conditions holds;

(i) $\max \left\{\alpha_{1}, \alpha_{2}\right\} \geq N+\gamma_{+}$.

(ii) $u_{0} \in H_{a_{1}}$ with $a_{1}<\alpha_{1}$ or $v_{0} \in H_{a_{2}}$ with $a_{2}<\alpha_{2}$.

Then, there exists no nontrivial nonnegative global solution of (1).

It is expected that if (6) holds, $\max \left\{\hat{\alpha}_{1}, \hat{\alpha}_{2}\right\}<N+\gamma_{+}, u_{0} \leq c\langle x\rangle^{-a_{1}} \psi_{1}(x /|x|)$ and $v_{0} \leq c\langle x\rangle^{-a_{2}} \psi_{1}(x /|x|)$ with $c>0$ small enough, $a_{i}>\hat{\alpha}_{i}(i=1,2)$, then the solution of $(1)$ is global in time, where $\hat{\alpha}_{i}=\left\{\left(2+\hat{\sigma}_{i}+2 \hat{q}_{i}\right)+\left(2+\hat{\sigma}_{j}+2 \hat{q}_{j}\right) p_{i}\right\} /$ $\left(p_{i} p_{j}-1\right)((i, j)=(1,2),(2,1))$. However, we have not proved it yet.

The method using the sequence of balls in (11) was used in [4, 22] and other papers.

REMARK. (i) It is easily seen that $\gamma_{+}=v_{1}-(N-2) / 2$.

(ii) If both (6) and (11) hold, then it is necessarily that $C_{U} \geq C_{L}, \hat{\sigma}_{i} \geq \check{\sigma}_{i}$ and $\hat{q}_{i} \geq \check{q}_{i}$.

We briefly recall a history of the study on global nonexistence of solutions to the system (1). First, the global nonexistence of solutions in the case $D=\mathbf{R}^{N}$ $\left(\Omega=S^{N-1}\right), u=v, p_{i}=p$ and $K_{i}(x, t)=1(i=1,2)$, that is

$$
\begin{cases}u_{t}=\Delta u+u^{p}, & x \in \mathbf{R}^{N}, t>0, \\ u(x, 0)=u_{0}(x) \geq 0, & x \in \mathbf{R}^{N},\end{cases}
$$

was studied by Fujita [3]. Fujita proved that when $p<1+2 / N$ the solution of (13) is not global in time for any nonnegative bounded and continuous initial data $u_{0} \not \equiv 0$. Fujita's results were also extended by some researcher. Hayakawa [8], Kobayashi-Sirao-Tanaka [11] and Weissler [28] proved that when $p=1+2 / N$, the solution of (13) blows up in finite time for any $u_{0} \not \equiv 0$. For the case $p>1+2 / N$, Lee-Ni [12] studied that if $\left\|u_{0}\right\|_{\infty}$ is large enough or $\liminf |x| \rightarrow \infty|x|^{a} u_{0}(x)>0$ with $a<2 /(p-1)$, the solution of (13) is not global in time. When $D$ is a cone, that is

$$
\begin{cases}u_{t}=\Delta u+u^{p}, & x \in D, t>0, \\ u(x, t)=0, & x \in \partial D, t>0, \\ u(x, 0)=u_{0}(x) \geq 0, & x \in D,\end{cases}
$$

Levine-Meier [14], [15] proved that if $p \leq 1+2 /\left(N+\gamma_{+}\right)$, there is no global solution of (14). 
Fujita's results were extended to the case $D=\mathbf{R}^{N}, u=v, p_{i}=p$ and $K_{i}(x, t)$ $=K(x, t)$ for $i=1,2$, that is

$$
\begin{cases}u_{t}=\Delta u+K(x, t) u^{p}, & x \in \mathbf{R}^{N}, t>0 \\ u(x, 0)=u_{0}(x) \geq 0, & x \in \mathbf{R}^{N}\end{cases}
$$

In the case $K(x, t)=|x|^{\sigma}$ with $\sigma \geq 0$, Bandle-Levine [1] had that when $p<1+$ $(2+\sigma) / N$ the solution of $(15)$ is not global in time for any $u_{0} \not \equiv 0$. Hamada [6] had the same result for $p=1+(2+\sigma) / N$ (see also [18]). Suzuki [23] extended to the case $\sigma \in \mathbf{R}$ for the quasilinear parabolic equations. Thereafter, Qi [20] extended the result to the case $K(x, t)=t^{q}|x|$ with $q \geq 0, \sigma \geq 0$. He proved that when $p \leq 1+(2+\sigma+2 q) / N$ there exists no global solution of (15). When $D$ is a cone, that is

$$
\begin{cases}u_{t}=\Delta u+K(x, t) u^{p}, & x \in D, t>0 \\ u(x, t)=0, & x \in \partial D, t>0 \\ u(x, 0)=u_{0}(x) \geq 0, & x \in D\end{cases}
$$

in the case $K(x, t)=|x|^{\sigma}$ with $\sigma \geq 0$, Levine-Meier [14], [15] and Hamada [6] had that if $p \leq 1+(2+\sigma) /\left(N+\gamma_{+}\right)$, there is no global solution of (16). For the case $p>1+(2+\sigma) /\left(N+\gamma_{+}\right)$, Hamada [7] studied that if $u_{0}(x) \geq M\langle x\rangle^{-a} \psi_{1}(x /|x|)$ with $a \leq(2+\sigma) /(p-1), 0 \leq \sigma \leq(p-1)(N-2)$ and some $M>0$, the solution of (16) is not global. In the case $K(x, t) \sim t^{q}$ with $q>-1$ as $t \rightarrow \infty$, LevineMeier [15] had that if $p \leq 1+(2+2 q) /\left(N+\gamma_{+}\right)$, there exists no global solution of (16).

In the case $D=\mathbf{R}^{N}$, our results are reduced to Escobedo-Herrero [2] and Mochizuki [16] with $K_{i}(x, t)=1(i=1,2)$, to Uda [24] with $K_{i}(x, t)=t^{q_{i}}$ $(i=1,2)$, and to Mochizuki-Huang [17] with $K_{i}(x, t)=|x|^{\sigma_{i}}$ with $\sigma_{i} \in$ $\left[0, n\left(p_{i}-1\right)\right)(i=1,2)$. Additionally, Guedda-Kirane [5] and Kirane-Qafsaoui [10] studied in this field. They studied the case $K_{i}(x, t) \sim t^{q_{i}}|x|^{\sigma_{i}}$ as $t \rightarrow \infty$ and $|x| \rightarrow \infty$. However, they needed the condition $\max \left\{2 q_{i}, \sigma_{i}\right\}<n\left(p_{i}-1\right)(i=1,2)$. Moreover, when $K_{i}(x, t)(i=1,2)$ satisfy (6) and (11) with $D=\mathbf{R}^{N}$, the system (1) was studied by Igarashi-Umeda [9]. When $D$ is a cone, in the case $K_{i}(x, t)=1$, the condition (i) of Theorem 2 is reduced to Levine [13].

The rest of the paper is organized as follows. In section 2, we state the proof of the local existence (Theorem 1). The proof of global nonexistence (Theorem 2) is given in section 3. For the change of variable as (3), we decide $\eta(x, y, t)=$ $\eta(r, \theta, \rho, \phi, t), \eta(x, t)=\eta(r, \theta, t)$ or $\eta_{0}(x)=\eta_{0}(r, \theta)$ for any function. 


\section{Local Existence in Time}

In this section we use $\sigma_{i}=\hat{\sigma}_{i}$ and $q_{i}=\hat{q}_{i}$ for $i=1,2$. In order to show the local solvability of the Cauchy problem (1), we consider the associated integral system

$$
\begin{aligned}
& u(x, t)=S(t) u_{0}(x)+\int_{0}^{t} S(t-s) K_{1}(x, s) v(x, s)_{1}^{p} d s, \\
& v(x, t)=S(t) v_{0}(x)+\int_{0}^{t} S(t-s) K_{2}(x, s) u(x, s)_{2}^{p} d s,
\end{aligned}
$$

where

$$
S(t) \xi(x)=\int_{D} G(x, y, t) \xi(y) d y
$$

with $G$ defined by (4). Define

$$
\Psi(u, v)=\left(S(t) u_{0}(x)+\Phi_{1}(v), S(t) v_{0}(x)+\Phi_{2}(u)\right),
$$

where

$$
\left(\Phi_{1}(v), \Phi_{2}(u)\right)=\int_{0}^{t} S(t-s)\left(K_{1}(x, s) v(x, s)^{p_{1}}, K_{2}(x, s) u(x, s)^{p_{2}}\right) d s .
$$

Lemma 2.1. Let $\delta \geq 0$ and $\alpha:=\max \{0,-\delta(N-2-\delta) / 2\}$. If we take $0<T \leq(\log 2) / \alpha$, then for $0 \leq t<T$

$$
\|S(t) \xi\|_{\infty, \delta} \leq 2\|\xi\|_{\infty, \delta} .
$$

Moreover for $0 \leq t<T$

$$
\left\|S(t)\langle\cdot\rangle^{-\delta}\right\|_{\infty, \delta} \leq 2
$$

Proof. Let $w(x, t):=S(t) \xi(x)-\|\xi\|_{\infty, \delta}\langle x\rangle^{-\delta} \exp (\alpha t)$, then we have

$$
\begin{aligned}
\Delta w-w_{t} & =\left[\alpha|x|^{4}+\{2 \alpha+\delta(N-2-\delta)\}|x|^{2}+N \delta+\alpha\right]\|\xi\|_{\infty, \delta}\langle x\rangle^{-\delta-4} \exp (\alpha t) \\
& \geq 0 .
\end{aligned}
$$

Combining this with Protter-Weinberger [19, Theorem 10, pp. 183-184], we get $w(x, t) \leq 0$; that is,

$$
\langle x\rangle^{\delta} S(t) \xi(x) \leq\|\xi\|_{\infty, \delta} \exp (\alpha t)
$$


Then we obtain $\|S(t) \xi\|_{\infty, \delta} \leq\|\xi\|_{\infty, \delta} \exp (\alpha t)$. Moreover, if we take $0<T \leq$ $(\log 2) / \alpha$, then for $0<t<T$

$$
\|S(t) \xi\|_{\infty, \delta} \leq 2\|\xi\|_{\infty, \delta}
$$

Lemma 2.2. (i) Assume that $\langle x\rangle^{\delta_{1}} u_{0}(x)$ and $\langle x\rangle^{\delta_{2}} v_{0}(x)$ are bounded in $\bar{D}$. $\left(S(\cdot) u_{0}, S(\cdot) v_{0}\right) \in E_{T}$ for $0<T \leq(\log 2) / \alpha$, and we have

$$
\left\|\left(S(\cdot) u_{0}, S(\cdot) v_{0}\right)\right\|_{E_{T}} \leq 2\left\{\left\|u_{0}\right\|_{\infty, \delta_{1}}+\left\|v_{0}\right\|_{\infty, \delta_{2}}\right\}
$$

where $E_{T}$ is defined in (7).

(ii) Let $(u, v) \in E_{T}$. Suppose that $K_{i}(x, t)(i=1,2)$ satisfy (6). Then $\left(\Phi_{1}(v), \Phi_{2}(u)\right) \in E_{T}$ for some $T>0$, and we have

$$
\left\|\left(\Phi_{1}(v), \Phi_{2}(u)\right)\right\|_{E_{T}} \leq 2 C_{U}\left(\tilde{T}_{1}(T)+\tilde{T}_{2}(T)\right)\left\{\|(0, v)\|_{E_{T}}^{p_{1}}+\|(u, 0)\|_{E_{T}}^{p_{2}}\right\}
$$

where the constant $C_{U}$ is appeared in $(6)$, and $\tilde{T}_{i}(t)=\left\{(t+1)^{q_{i}+1}-1\right\} /\left(q_{i}+1\right)$ $(i=1,2)$.

Proof. (i) It is obvious from Lemma 2.1 with $\delta=\delta_{i}(i=1,2)$.

(ii) Note that

$$
\begin{aligned}
& \int_{0}^{t} S(t-s) K_{1}(x, s) v(x, s)^{p_{1}} d s \\
& \quad \leq \int_{0}^{t} S(t-s) C_{U}(s+1)^{q_{1}}\langle x\rangle^{\sigma_{1}-\delta_{2} p_{1}} d s \sup _{s \in[0, t]}\|v(\cdot, s)\|_{\infty, \delta_{2}}^{p_{1}} .
\end{aligned}
$$

A simple calculation gives $-\sigma_{1}+\delta_{2} p_{1}=\delta_{1}$. Then it follows from Lemma 2.1 that

$$
\left\|S(t-s)\langle\cdot\rangle^{\sigma_{1}-\delta_{2} p_{1}}\right\|_{\infty, \delta_{1}} \leq 2
$$

Thus we have

$$
\left\|\Phi_{1}(v)\right\|_{\infty, \delta_{1}} \leq 2 C_{U} \tilde{T}_{1}(t) \sup _{s \in[0, t]}\|v(s)\|_{\infty, \delta_{2}}^{p_{1}}
$$

Similarly, we have

$$
\left\|\Phi_{2}(u)\right\|_{\infty, \delta_{2}} \leq 2 C_{U} \tilde{T}_{2}(t) \sup _{s \in[0, t]}\|u(s)\|_{\infty, \delta_{1}}^{p_{2}}
$$

We conclude from these inequations. 
Proof OF Theorem 1. Let $B_{R}=\left\{(u, v) \in E_{T} ;\|(u, v)\|_{E_{T}} \leq R\right\}$ and $P_{T}=$ $\left\{(u, v) \in E_{T} ; u \geq 0, v \geq 0\right\}$, and define $\tilde{T}_{i}$ same as in Lemma 2.2 (ii). For $\left(u_{1}, v_{1}\right)$, $\left(u_{2}, v_{2}\right) \in B_{R} \cap P_{T}$ with $R \geq 1$ sufficient large, we have

$$
\left\|\Psi\left(u_{1}, v_{1}\right)-\Psi\left(u_{2}, v_{2}\right)\right\|_{E_{T}}=\left\|\left(\Phi_{1}\left(v_{1}\right)-\Phi_{1}\left(v_{2}\right), \Phi_{2}\left(u_{1}\right)-\Phi_{2}\left(u_{2}\right)\right)\right\|_{E_{T}} .
$$

We consider

$$
\begin{aligned}
& \left|\Phi_{1}\left(v_{1}\right)-\Phi_{1}\left(v_{2}\right)\right|\langle x\rangle^{\delta_{1}} \\
& \quad \leq \int_{0}^{t} S(t-s) C_{U}(s+1)^{q_{1}}\langle x\rangle^{\sigma_{1}}\left|v_{1}(x, s)^{p_{1}}-v_{2}(x, s)^{p_{1}}\right| d s\langle x\rangle^{\delta_{1}} .
\end{aligned}
$$

Then, since $\left(u_{2}, v_{2}\right) \in B_{R}$ we obtain

$$
\begin{aligned}
& \left|\Phi_{1}\left(v_{1}\right)-\Phi_{1}\left(v_{2}\right)\right|\langle x\rangle^{\delta_{1}} \\
& \quad \leq 2^{p_{1}} C_{U} \tilde{T}_{1}(T) \sup _{s \in[0, t]}\left\|R^{p_{1}-1} p_{1}\left(v_{1}(\cdot, s)-v_{2}(\cdot, s)\right)\right\|_{\infty, \delta_{2}} .
\end{aligned}
$$

By the same argument we have

$$
\begin{aligned}
& \left|\Phi_{2}\left(u_{1}\right)-\Phi_{2}\left(u_{2}\right)\right|\langle x\rangle^{\delta_{2}} \\
& \quad \leq 2^{p_{2}} C_{U} \tilde{T}_{2}(T) \sup _{s \in[0, t]}\left\|R^{p_{2}-1} p_{2}\left(u_{1}(\cdot, s)-u_{2}(\cdot, s)\right)\right\|_{\infty, \delta_{1}} .
\end{aligned}
$$

Substitute (22) and (23) into (21). Since $\max \left\{p_{1}, p_{2}\right\} \leq p_{1} p_{2}$, we obtain

$$
\begin{aligned}
& \left\|\Psi\left(u_{1}, v_{1}\right)-\Psi\left(u_{2}, v_{2}\right)\right\|_{E_{T}} \\
& \quad \leq 2^{p_{1} p_{2}} C_{U}\left(\tilde{T}_{1}(T)+\tilde{T}_{2}(T)\right) R^{p_{1} p_{2}-1} p_{1} p_{2}\left\|\left(u_{1}-u_{2}, v_{1}-v_{2}\right)\right\|_{E_{T}} .
\end{aligned}
$$

Taking $T>0$ small enough, we have

$$
\left\|\Psi\left(u_{1}, v_{1}\right)-\Psi\left(u_{2}, v_{2}\right)\right\|_{E_{T}} \leq \rho\left\|\left(u_{1}, v_{1}\right)-\left(u_{2}, v_{2}\right)\right\|_{E_{T}}
$$

for some $\rho<1$. Then $\Psi$ is a strict contraction of $B_{R} \cap P_{T}$ into itself, whence there exists a unique fixed point $(u, v) \in B_{R} \cap P_{T}$ which solves (1).

\section{Nonexistence of Global Solution}

In this section we treat the nonexistence of global solutions in time of (1). Here, we take the same strategy as in [17], [18], [25] and [26]. Let $\sigma_{i}=\check{\sigma}_{i}$ and $q_{i}=\check{q}_{i}$ for $i=1,2$ through this section.

First, we should consider only the case $k \in(0,1 / 2)$ by comparison. Let 
$\lambda_{m}>0$ denote the principal eigenvalue of $-\Delta$ with Dirichlet problem in $B\left(x_{m}, k\left|x_{m}\right|\right)$, and let $\zeta_{m}(x)>0$ denote the corresponding positive eigenfunction, normalized by $\int_{B\left(x_{m}, k\left|x_{m}\right|\right)} \zeta_{m}(x) d x=1$. Define

$$
F_{m}(t)=\int_{B\left(x_{m}, k\left|x_{m}\right|\right)} u(x, t) \zeta_{m}(x) d x, \quad G_{m}(t)=\int_{B\left(x_{m}, k\left|x_{m}\right|\right)} v(x, t) \zeta_{m}(x) d x .
$$

By applying Green's formula and Jensen's inequality, we see that $\left(F_{m}(t), G_{m}(t)\right)$ satisfies

$$
\left\{\begin{array}{l}
F_{m}^{\prime}(t) \geq-c_{1}\left|x_{m}\right|^{-2} F_{m}(t)+c_{2} t^{q_{1}}\left|x_{m}\right|^{\sigma_{1}} G_{m}(t)^{p_{1}}, \\
G_{m}^{\prime}(t) \geq-c_{1}\left|x_{m}\right|^{-2} G_{m}(t)+c_{2} t^{q_{2}}\left|x_{m}\right|^{\sigma_{2}} F_{m}(t)^{p_{2}}
\end{array}\right.
$$

(see $[9, \S 3])$. We will show that for an appropriate choice of $k,\left(F_{m}(t), G_{m}(t)\right)$ is not global in time, thereby contradicting the assumption that $(u, v)$ is a global solution. By the same arguments as in $[9, \S 3],[13]$ and [21], we have the following proposition:

Proposition 3.1. Let $\left(F_{m}(t), G_{m}(t)\right)$ by $(24)$ for some $t_{0} \in(0, t]$ and $m \in \mathbf{N}$. If

$$
F_{m}\left(c_{1}\left|x_{m}\right|^{2}\right)>A\left|x_{m}\right|^{-\alpha_{1}} \text { or } G_{m}\left(c_{1}\left|x_{m}\right|^{2}\right)>B\left|x_{m}\right|^{-\alpha_{2}}
$$

with some $A, B>0$ and some $c_{1}>0$, then $\left(F_{m}(t), G_{m}(t)\right)$ is not global in time.

Lemma 3.1. Let $u_{0}$ and $v_{0}$ are $B C$ and $\left(u_{0}, v_{0}\right) \not \equiv 0$, and let $(u, v)$ be a solution of (1). Then for any $\tau>0$ and $x \in D$ there exist constants $\mu \geq 1$ and $C=$ $C\left(N, \tau, u_{0}, v_{0}, K_{1}, K_{2}, p_{1}, p_{2}, \mu\right)>0$ such that

$$
u(x, \tau) \geq C|x|^{\gamma_{+}} e^{-\mu|x|^{2}} \psi_{1}(x /|x|) \quad \text { and } \quad v(x, \tau) \geq C|x|^{\gamma_{+}} e^{-\mu|x|^{2}} \psi_{1}(x /|x|) .
$$

Proof. We may let $u_{0}(x) \not \equiv 0$ without loss of generality. Since $u(x, t) \geq$ $S(t) u_{0}(x), I_{v}(z) \geq C z^{v}$ and $\gamma_{+}=v_{1}-(N-2) / 2$, we obtain

$$
\begin{aligned}
u(x, t) \geq & \frac{C}{(2 t)^{1+v_{1}}} r^{\gamma_{+}} e^{-r^{2} / 4 t} \psi_{1}(\theta) \\
& \times \int_{0}^{\infty} \int_{\Omega} \rho^{\gamma_{+}+N-1} e^{-\rho^{2} / 4 t} \psi_{1}(\phi) u_{0}(\rho, \phi) d \phi d \rho .
\end{aligned}
$$

Then we have, for every $\tau_{1}>0$,

$$
u\left(x, \tau_{1}\right) \geq C_{1} r^{\gamma_{+}} e^{-\mu_{1} r^{2}} \psi_{1}(\theta)
$$

with $\mu_{1}=\max \left\{1,1 / 4 \tau_{1}\right\}$ and 


$$
C_{1}=C_{1}\left(\tau_{1}, N, u_{0}\right)=\frac{C}{\left(2 \tau_{1}\right)^{1+v_{1}}} \int_{0}^{\infty} \int_{\Omega} \rho^{\gamma_{+}+N-1} e^{-\rho^{2} / 4 \tau_{1}} \psi_{1}(\phi) u_{0}(\rho, \phi) d \phi d \rho
$$

From (18) and the fact that $I_{v} \geq C z^{v}$ with some $C>0$, we have

$$
\begin{aligned}
v(x, t) \geq & C r^{\gamma_{+}} \psi_{1}(\theta) \int_{0}^{t} \int_{0}^{\infty} \int_{\Omega} \frac{1}{(2(t-s))^{v_{1}+1}} e^{-r^{2} / 4(t-s)} \\
& \times \rho^{\gamma_{+}+N-1} e^{-\rho^{2} / 4(t-s)} \psi_{1}(\phi) K_{2}(\rho, \phi, s) u^{p_{2}}(\rho, \phi, s) d \phi d \rho d s .
\end{aligned}
$$

Then by (25) we obtain for $\tau_{2}>2 \tau_{1}$

$$
\begin{aligned}
v\left(x, \tau_{2}\right) & \geq C_{2} r^{\gamma_{+}} \psi_{1}(\theta) \int_{\tau_{2} / 2}^{\tau_{2}} \frac{1}{\left(2\left(\tau_{2}-s\right)\right)^{v_{1}+1}} e^{-r^{2} / 4\left(\tau_{2}-s\right)} d s \\
& \geq C_{2} r^{\gamma_{+}} \psi_{1}(\theta) \frac{1}{\tau_{2}^{\nu_{1}+1}} e^{-r^{2} / 2 \tau_{2}} \int_{\tau_{2} / 2}^{\tau_{2}} d s=C_{2} r^{\gamma_{+}} \psi_{1}(\theta) \frac{1}{2 \tau_{2}^{\nu_{1}}} e^{-r^{2} / 2 \tau_{2}}
\end{aligned}
$$

with

$$
\begin{aligned}
C_{2} & =C_{2}\left(\tau_{2}, N, u_{0}, K_{2}\right) \\
& =\inf _{s \in\left(\tau_{2} / 2, \tau_{2}\right)} C \int_{0}^{\infty} \int_{\Omega} \rho^{\gamma_{+}+N-1} e^{-\rho^{2} / 4\left(\tau_{2}-s\right)} \psi_{1}(\phi) K_{2}(\rho, \phi, s) u^{p_{2}}(\rho, \phi, s) d \phi d \rho .
\end{aligned}
$$

Then we have

$$
v\left(x, \tau_{2}\right) \geq C_{3} r^{\gamma_{+}} e^{-\mu_{2} r^{2}} \psi_{1}(\theta)
$$

with $\mu_{2}=\max \left\{1,1 / 2 \tau_{2}\right\}$ and $C_{3}=C_{2} / 2 \tau_{2}^{\nu_{1}}$. Put $C=\min \left\{C_{1}, C_{2}, C_{3}\right\}$ and $\mu=$ $\max \left\{\mu_{1}, \mu_{2}\right\}$ and $\tau=\tau_{2}$. Then we have

$$
u(x, \tau) \geq C r^{\gamma} e^{-\mu r^{2}} \psi_{1}(\theta) \text { and } v(x, \tau) \geq C r^{\gamma_{+}} e^{-\mu r^{2}} \psi_{1}(\theta) .
$$

Lemma 3.2. For $\sigma \geq 0, \mu \geq 1, x \in D$ and $t \geq \tau$ with some $\tau>0$, we have

$$
S(t) \chi_{B}(x)|x|^{\sigma} e^{-\mu|x|^{2}} \geq C t^{\left(\sigma-\gamma_{+}\right) / 2}(1+4 \mu t)^{-\left(N+\sigma+\gamma_{+}\right) / 2}|x|^{\gamma_{+}} e^{-|x|^{2} / 4 t} \psi_{1}(x /|x|)
$$

with some $C>0$ and $B=B(b, a) \subset D$ with $a>0$ and $b \in D$, where $\chi_{B}$ is $a$ characteristic function of $B$ such that $\chi_{B}(x)=1$ for $x \in B$ and $=0$ for $x \in D \backslash B$. The domain $B(b, a)$ denotes the open ball of radius a centered at $b$.

Proof. We can put positive constants $a_{1}, a_{2}$ and domain $\Omega^{\prime} \subset \Omega$ satisfying $0<a_{1}<a_{2}<\infty, \quad \Omega^{\prime} \mid \neq 0$ and $D_{B}=\left\{x ;|x| \in\left(a_{1}, a_{2}\right), x /|x| \in \Omega^{\prime}\right\} \subset B$. By (19) and $\int_{\Omega^{\prime}} \psi_{1}(\phi) d \phi=C$ with some $C \in(0,1]$, we have 


$$
\begin{aligned}
& S(t) \chi_{B}(x)|x|^{\sigma} e^{-v|x|^{2}} \\
& \quad \geq \int_{a_{1}}^{a_{2}} \int_{\Omega^{\prime}} G(r, \theta, \rho, \phi, t) \rho^{\sigma} e^{-\mu \rho^{2}} \rho^{N-1} d \phi d \rho \\
& \quad \geq \frac{C}{(2 t)^{1+v_{1}}} r^{\gamma_{+}} e^{-r^{2} / 4 t} \psi_{1}(\theta) \int_{a_{1}}^{a_{2}} \int_{\Omega^{\prime}} \rho^{\gamma_{+}+\sigma+N-1} e^{-(1+4 \mu t) \rho^{2} / 4 t} \psi_{1}(\phi) d \phi d \rho \\
& \quad \geq \frac{C r^{\gamma} e^{-r^{2} / 4 t} \psi_{1}(\theta)}{(2 t)^{1+v_{1}} \tilde{\mu}(t)^{\gamma_{+}+\sigma+N}} \int_{\tilde{\mu}(t) a_{1}}^{\tilde{\mu}(t) a_{2}} s^{\gamma_{+}+\sigma+N-1} e^{-s^{2}} d s
\end{aligned}
$$

where $\tilde{\mu}(t)=\sqrt{(1+4 \mu t) / 4 t}$. Since $1 \leq \sqrt{\mu} \leq \tilde{\mu}(t) \leq \tilde{\mu}(\tau)$ for $t \geq \tau$, we have

$$
S(t) \chi_{B}(x)|x|^{\sigma} e^{-v|x|^{2}} \geq C t^{\left(\sigma-\gamma_{+}\right) / 2}(1+4 \mu t)^{-\left(N+\sigma+\gamma_{+}\right) / 2} r^{\gamma_{+}} e^{-r^{2} / 4 t} \psi_{1}(\theta) .
$$

By Lemma 3.1, we can assume

$$
u_{0}(x) \geq C|x|^{\gamma_{+}} e^{-\mu|x|^{2}} \psi_{1}(x /|x|)
$$

for some $C>0$ and $\mu>0$. Then we have, for $t \geq \tau$

$$
u(x, t) \geq C(1+4 \mu t)^{-N / 2-\gamma_{+}}|x|^{\gamma_{+}} e^{-|x|^{2} / 4 t} \psi_{1}(x /|x|) .
$$

Lemma 3.3. Let $v$ be the second element of the solution of (1). Then we have

$$
v(x, t) \geq C t^{\left(\left(p_{2}-1\right) \gamma_{+}+\sigma_{2}+2 q_{2}+2\right) / 2}(t+1)^{-\gamma_{+} p_{2}-N p_{2} / 2}|x|^{\gamma_{+}} e^{-|x|^{2} / 2 t} \psi(x /|x|)^{p_{2}+1}
$$

for $t \geq \tau$ with some $\tau>0$ and $C=C\left(\tau, u_{0}, v_{0}, K_{1}, K_{2}, p_{1}, p_{2}\right)>0$.

Proof. It follows from (11), (18) and (26), we obtain

$$
\begin{aligned}
v(x, t) \geq & C \int_{0}^{t} S(t-s) \chi_{\tilde{B}_{k, 1}}(x)|x|^{\sigma_{2}+p_{2} \gamma_{+}} s^{q_{2}} \\
& \times(4 s+1 / \mu)^{-N p_{2} / 2-p_{2} \gamma_{+}} e^{-p_{2}|x|^{2} / 4 s} \psi_{1}^{p_{2}}(x /|x|) d s .
\end{aligned}
$$

By Lemma 3.2, we then have

$$
\begin{aligned}
v(x, t) \geq & C(t / 2)^{\left(p_{2}-1\right) \gamma_{+} / 2+\sigma_{2} / 2}(t / 4)^{q_{2}}(2 t+1 / \mu)^{-\gamma_{+} p_{2}-N p_{2} / 2} \\
& \times|x|^{\gamma_{+}} e^{-|x|^{2} / 2 t} \psi_{1}^{p_{2}+1}(x /|x|) \int_{t / 4}^{t / 2} d s .
\end{aligned}
$$

Thus, the inequality of the lemma holds. 
Lemma 3.4. Let $u$ be first element of the solution of (1) and $\alpha_{1} \geq N+\gamma_{+}$. Then for $t \geq a$

$$
u(x, t) \geq\left\{\begin{array}{l}
C t^{-N / 2-\gamma_{+}}|x|^{\gamma_{+}} e^{-|x|^{2} / 2 t} \psi_{1}(x /|x|)^{p_{1} p_{2}+p_{1}+1} \log (t / 2 a), \quad \text { if } \alpha_{1}=N+\gamma_{+}, \\
C t^{-N / 2-\gamma_{+}}|x|^{\gamma_{+}} e^{-|x|^{2} / 2 t} \psi_{1}(x /|x|)^{p_{1} p_{2}+p_{1}+1}\left(t^{\tilde{p}}-(2 a)^{\tilde{p}}\right), \quad \text { if } \alpha_{1}>N+\gamma_{+}
\end{array}\right.
$$

with $C=C\left(a, u_{0}, v_{0}, K_{1}, K_{2}, p_{1}, p_{2}, N\right)>0$, where $a>0$ is a small constant and $\tilde{p}=\left(p_{1} p_{2}-1\right)\left(\alpha_{1}-N-\gamma_{+}\right) / 2$.

Proof. By Lemma 3.3, we have

$$
\begin{aligned}
u(x, t) \geq & C \int_{a}^{t} S(t-s) \chi_{\tilde{B}_{k, 1}}(x)|x|^{\sigma_{1}} s^{q_{1}} s^{\left(1+\sigma_{2} / 2+q_{2}\right) p_{1}+p_{1}\left(p_{2}-1\right) \gamma_{+} / 2} \\
& \times(s+1)^{-N p_{1} p_{2} / 2-\gamma_{+} p_{1} p_{2}}|x|^{p_{1} \gamma_{+}} e^{-p_{1}|x|^{2} / 2 s} \psi_{1}(x /|x|)^{p_{1}\left(p_{2}+1\right)} d s .
\end{aligned}
$$

It follows from Lemma 3.2 that

$$
\begin{aligned}
u(x, t) \geq & C(t / 2)^{\left(\sigma_{1}+p_{1} \gamma_{+}-\gamma_{+}\right) / 2} t^{-\left(N+\sigma_{1}+p_{1} \gamma_{+}+\gamma_{+}\right) / 2}|x|^{\gamma_{+}} e^{-|x|^{2} / 2 t} \\
& \times \psi_{1}(x /|x|)^{p_{1} p_{2}+p_{1}+1} \int_{a}^{t / 2} s^{\left\{\left(-N-\gamma_{+}\right)\left(p_{1} p_{2}-1\right)+\left(2+\sigma_{2}+2 q_{2}\right) p_{1}+\sigma_{1}+2 q_{1}\right\} / 2} d s
\end{aligned}
$$

for small $a>0$. Since

$$
\begin{aligned}
& \left\{\left(-N-\gamma_{+}\right)\left(p_{1} p_{2}-1\right)+\left(2+\sigma_{2}+2 q_{2}\right) p_{1}+\sigma_{1}+2 q_{1}\right\} / 2 \\
& \quad=\left\{\left(p_{1} p_{2}-1\right)\left(\alpha_{1}-N-\gamma_{+}\right)\right\} / 2-1,
\end{aligned}
$$

this proves the inequality of the lemma.

Proof of TheOrem 2. First we consider the case (i). We may assume $\alpha_{1} \geq \alpha_{2}$. Put $Y_{m}=\sqrt{c_{1}}\left|x_{m}\right|$. From the definition, we have $\alpha_{1} \geq N+\gamma_{+}$. By Lemma 3.4, since $x \in B\left(x_{m}, k\left|x_{m}\right|\right)$, we have

$$
\begin{aligned}
F_{m}\left(Y_{m}^{2}\right) \geq & C Y_{m}^{-N-\gamma_{+}} h_{m} \\
& \times \int_{B\left(x_{m}, k\left|x_{m}\right|\right)} \frac{|x|^{\gamma_{+}}}{\left|x_{m}\right|^{\gamma_{+}}} \exp \left(-\frac{|x|^{2}}{2 Y_{m}^{2}}\right) \zeta_{m}(x) \psi_{1}(x /|x|)^{p_{1} p_{2}+p_{1}+1} d x \\
\geq & C\left|x_{m}\right|^{-N-\gamma_{+}}\left(c_{1}\right)^{-\left(N+\gamma_{+}\right) / 2} h_{m}(1+k)^{\gamma_{+}} \exp \left(-(1+k)^{2} / 2 c_{1}\right),
\end{aligned}
$$


where $h_{m}=\log \left(Y_{m}^{2} / 2 a\right)$ for $\alpha_{1}=N+\gamma_{+}$and $h_{m}=Y_{m}^{2 \tilde{p}}-(2 a)^{\tilde{p}}$ for $\alpha_{1}>N+\gamma_{+}$ with $C=C\left(a, u_{0}, v_{0}, K_{1}, K_{2}, p_{1}, p_{2}, N\right)>0$ and $\tilde{p}$ defined in Lemma 3.4. Since $\alpha_{1} \geq N+\gamma_{+}$, it follows that

$$
\begin{aligned}
\left|x_{m}\right|^{\alpha_{1}} F_{m}\left(Y_{m}^{2}\right) \geq & C\left|x_{m}\right|^{\alpha_{1}-N-\gamma_{+}} c_{1}^{-\left(N+\gamma_{+}\right) / 2} \\
& \times h_{m}(1+k)^{\gamma_{+}} \exp \left(-(1+k)^{2} / 2 c_{1}\right)>A
\end{aligned}
$$

for $m$ large enough. Thus, $\left(F_{m}(t), G_{m}(t)\right)$ is not global in time by Proposition 3.1.

Next, we consider the case (ii). Since $u(x, t) \geq S(t) u_{0}(x), u \in H_{a_{1}}, I_{v}(z) \geq C z^{v}$ and $\int_{\Omega} \psi_{1}(\phi)^{2} d \phi$ is constant, it follows that

$$
\begin{aligned}
u(x, t) & \geq \int_{0}^{\infty} \int_{\Omega} G(r, \theta, \rho, \phi, t) u_{0}(\rho, \phi) \rho^{N-1} d \phi d \rho \\
& \geq C \int_{0}^{\infty} \frac{r^{\gamma_{+}} \rho^{N+\gamma_{+}-1}}{t^{\gamma_{+}+N / 2}} \exp \left(-\frac{r^{2}+\rho^{2}}{4 t}\right)\left(1+\rho^{2}\right)^{-a_{1} / 2} \psi_{1}(\theta) d \rho .
\end{aligned}
$$

Then, since $\gamma_{+}=v_{1}-(N-2) / 2$, we obtain

$$
\begin{aligned}
u\left(x, Y_{m}^{2}\right) \geq & C\left(\frac{r}{Y_{m}}\right)^{\gamma_{+}} \exp \left(-\frac{r^{2}}{4 Y_{m}^{2}}\right) \int_{0}^{\infty} \frac{1}{Y_{m}}\left(\frac{\rho}{Y_{m}}\right)^{N / 2+v_{1}} \\
& \times \exp \left(-\frac{\rho^{2}}{4 Y_{m}^{2}}\right)\left(1+\rho^{2}\right)^{-a_{1} / 2} \psi_{1}(\theta) d \rho .
\end{aligned}
$$

Since $Y_{m}=\sqrt{c_{1}\left|x_{m}\right|^{2}}$, we have for $x \in B\left(x_{m}, k\left|x_{m}\right|\right)$

$$
\begin{aligned}
u\left(x, Y_{m}^{2}\right) \geq & C(1+k)^{-N / 2}(1-k)^{1+v_{1}} c_{1}^{-\gamma_{+} / 2} \exp \left\{-(1+k)^{2} / c_{1}\right\} \\
& \times \psi_{1}(\theta) \int_{0}^{\infty} \frac{1}{Y_{m}}\left(\frac{\rho}{Y_{m}}\right)^{N / 2+v_{1}} \exp \left(-\frac{\rho^{2}}{4 Y_{m}^{2}}\right)\left(1+\rho^{2}\right)^{-a_{1} / 2} d \rho .
\end{aligned}
$$

Putting $\chi=\rho / Y_{m}$, we have

$$
u\left(x, Y_{m}^{2}\right) \geq C \psi_{1}(\theta) \int_{0}^{\infty} \chi^{N / 2+v_{1}} \exp \left(-\frac{\chi^{2}}{4}\right)\left(1+\chi^{2} Y_{m}^{2}\right)^{-a_{1} / 2} d \chi
$$

Note that $1+\chi^{2} Y_{m}^{2} \leq Y_{m}^{2}\left(1+\chi^{2}\right)$ if $m$ is large enough. Then, we obtain

$$
\begin{aligned}
u\left(x, Y_{m}^{2}\right) & \geq C \psi_{1}(\theta)\left|Y_{m}\right|^{-a_{1}} \int_{0}^{\infty} \chi^{N / 2+v_{1}} \exp \left(-\frac{\chi^{2}}{4}\right)\left(1+\chi^{2}\right)^{-a_{1} / 2} d \chi \\
& \geq C \psi_{1}(x /|x|)\left|x_{m}\right|^{-a_{1}}
\end{aligned}
$$


for sufficiently large $m$. Since $\int_{B\left(x_{m}, k\left|x_{m}\right|\right)} \psi_{1}(x /|x|) \zeta_{m}(x) d x$ is constant, we have

$$
\begin{aligned}
F_{m}\left(Y_{m}^{2}\right) & \geq \int_{B\left(x_{m}, k\left|x_{m}\right|\right)} u\left(x, Y_{m}^{2}\right) \zeta_{m}(x) d x \\
& \geq C\left|x_{m}\right|^{-a_{1}} \int_{B\left(x_{m}, k\left|x_{m}\right|\right)} \psi_{1}(x /|x|) \zeta_{m}(x) d x \geq C\left|x_{m}\right|^{-a_{1}}
\end{aligned}
$$

Since $u_{0} \in H_{a_{1}}$ with $a_{1}<\alpha_{1}$, we have

$$
\left|x_{m}\right|^{\alpha_{1}} F_{m}\left(Y_{m}^{2}\right) \geq C\left|x_{m}\right|^{\alpha_{1}-a_{1}}>A
$$

for sufficiently large $m$. If $v_{0} \in H_{a_{2}}$ with $a_{2}<\alpha_{2}$, we similarly have

$$
\left|x_{m}\right|^{\alpha_{2}} G_{m}\left(Y_{m}^{2}\right) \geq C\left|x_{m}\right|^{\alpha_{2}-a_{2}}>B
$$

for $m$ large enough. Thus, $\left(F_{m}(t), G_{m}(t)\right)$ is not global in time by Proposition 3.1.

\section{Acknowledgement}

Much of the work of the second author was done while he visited the University of Tokyo during 2005-2008 as a postdoctoral fellow. Its hospitality is gratefully acknowledged as well as support from formation of $\mathrm{COE}$ "New Mathematical Development Center to Support Scientific Technology" (University of Tokyo), supported by JSPS.

\section{References}

[ 1 ] C. Bandle and H. A. Levine, On the existence and nonexistence of global solution of reactiondiffusion equation in sectorial domains, Trans. Amar. Math. Sec. 316 (1989), 595-622.

[2] M. Escobedo and M. A. Herrero, Boundness and blow up for a semilinear reaction-diffusion system, J. Diff. Eqns. 89 (1991), 176-202.

[ 3 ] H. Fujita, On the blowing up of solutions of the Cauchy problem for $u_{t}=\Delta u+u^{1+\alpha}$, J. Fac. Sci. Univ. Tokyo Sect. A Math. 16 (1966), 109-124.

[4] Y. Giga and N. Umeda, Blow-up directions at space infinity for solutions of semilinear heat equations, Bol. Soc. Parana. Mat. 23 (2005), 9-28.

[ 5] M. Guedda and M. Kirane, Criticality for some evolution equations, Differential Equations 37 (2001), 540-550.

[6] T. Hamada, Nonexistence of global solutions of parabolic equations in conical domains, Tsukuba J. Math. 19 (1995), 15-25.

[7] T. Hamada, On the existence and nonexistence of global solutions of semilinear parabolic equations with slowly decaying initial data, Tsukuba J. Math. 21 (1997), 505-514.

[ 8 ] K. Hayakawa, On nonexistence of global solution of some semilinear parabolic equations, Proc. Japan. Acad. 49 (1973), 503-505.

[ 9 ] T. Igarashi and N. Umeda, Existence and nonexistence of global solutions in time for a reactiondiffusion system with inhomogeneous terms, Funkcialaj Ekvacioj 51 (2008), 17-37. 
[10] M. Kirane and M. Qafsaoui, Global nonexistence for the Cauchy problem of some nonlinear reaction-diffusion systems, J. Math. Anal. Appli. 268 (2002), 217-243.

[11] K. Kobayashi, T. Sirao and H. Tanaka, On glowing up problem for semilinear heat equations, J. Math. Soc. Japan 29 (1977), 407-424.

[12] T.-Y. Lee and W.-M. Ni, Global existence, large time behavior and life span on solutions of semilinear Cauchy problem, Trans. Amer. Math. Soc. 333 (1992), 365-378.

[13] H. A. Levine, A Fujita type global existence-global nonexistence theorem for a weakly coupled system of reaction-diffusion equations, J. Appli. Math. Phys. (ZAMP) 42 (1991), 408 430.

[14] H. A. Levine and P. Meier, The value of critical exponent for reaction-diffusion equation in cones, Arch. Ratl. Mech. Anal. 109 (1990), 73-80.

[15] H. A. Levine and P. Meier, A blowup result for the critical exponent in cones, Israel J. Math. 67 (1989), 129-136.

[16] K. Mochizuki, Blow-up, life-span and large time behavior of solutions of a weakly coupled system of reaction-diffusion equations, Adv. Math. Appl. Sci. 48, World Scientific 1998, 175-198.

[17] K. Mochizuki and Q. Huang, Existence and behavior of solutions for a weakly coupled system of reaction-diffusion equations, Methods Appl. Anal. 5 (2) (1998), 109-124.

[18] R. G. Pinsky, Existence and nonexistence of global solutions for $u_{t}=\Delta u+a(x) u^{p}$ in $\mathbf{R}^{n}$, J. Differential Equations 133 (1997), 152-177.

[19] M. H. Protter and H. F. Weinberger, "Maximum principles in Differential Equations", PrenticeHall, Englewood Cliffs, New Jersey, 1967.

[20] Y.-W. Qi, The critical exponents of parabolic equations and blow-up in $\mathbf{R}^{n}$, Proc. Roy. Soc. Edinburgh Sect. 128A (1998), 123-136.

[21] Y.-W. Qi and H. A. Levine, The critical exponent of degenerate parabolic systems, Z. Angew Math. Phys. 44 (1993), 249-265.

[22] M. Shimojyo, On blow-up phenomenon at space infinity and its locality for semilinear heat equations (in Japanese), Master's Thesis, The University of Tokyo (2005).

[23] R. Suzuki, Existence and nonexistence of global solutions of quasilinear parabolic equations, J. Math. Soc. Japan, 54 (2002), 747-792.

[24] Y. Uda, The critical exponent for a weakly coupled system of the generalized Fujita type reaction-diffusion equations, Z. Angew Math. Phys. 46 (1995), 366-383.

[25] N. Umeda, Blow-up and large time behavior of solutions of a weakly coupled system of reaction-diffusion equations, Tsukuba J. Math. 27 (2003), 31-46.

[26] N. Umeda, Existence and nonexistence of global solutions of a weakly coupled system of reaction-diffusion equations, Comm. Appl. Anal. 10 (2006), 57-78.

[27] G. N. Watson, A Treatise on the Theory of Bessel Functions, 2nd Ed., Cambridge University Press, London/New York 1944.

[28] F. B. Weissler, Existence and nonexistence of global solutions for semilinear heat equation, Israel J. Math. 38 (1981), 29-40.

\section{Takefumi Igarashi}

College of Science and Technology, Nihon University

7-24-1, Narashino-dai, Funabashi-shi, Chiba, 274-8501, Japan

E-mail: igarashit@penta.ge.cst.nihon-u.ac.jp

Noriaki Umeda

Graduate School of Mathematical Sciences, University of Tokyo 3-8-1, Komaba, Meguro-ku, Tokyo, 153-8914, Japan

E-mail: umeda@ms.u-tokyo.ac.jp 